\title{
Epidemiological features and risk factors for mortality in Pseudomonas aeruginosa bacteremia in children
}

\author{
María T. Rosanova, M.D. ${ }^{a}$, María S. Mussini, M.D. ${ }^{a}$, Ana P. Arias, M.D. ${ }^{a}$, María I. Sormani, M.D. ${ }^{a}$, \\ Alejandra Mastroianni, Laboratory Technician ${ }^{b}$, Maria E. García, Biochemist ${ }^{b}$, Vanesa Reijtman, Biochemist ${ }^{b}$ \\ and Claudia Sarkis, M.D. ${ }^{a}$
}

\begin{abstract}
The objective was to describe the epidemiological, clinical, microbiological, and evolutionary characteristics and the risk factors for mortality.

Retrospective, cohort study. A total of 100 patients were included. Of these, $42(42 \%)$ had septic shock upon admission and $56(56 \%)$ were admitted to the intensive care unit. Bacteremia was primary in 17 patients $(17 \%)$; catheter-related, in $15(15 \%)$; and secondary, in $68(68 \%)$. The most common source of infection was the skin and mucous membrane. Resistance to one or more antibiotic groups was $38 \%$. Thirtyone patients died (31\%).

Risk factors for mortality were septic shock $(p<0.0005)$, admission to the intensive care unit $(p<0.0001)$, primary bacteremia $(p<0.009)$ or secondary, non-catheter-related bacteremia $(p<0.003)$, presence of mucocutaneous or pulmonary source of infection $(p<0.004)$, and multidrug resistance ( $p<$ $0.01)$ or resistance to carbapenems $(p<0.01)$.

Key words: bacteremia, Pseudomonas aeruginosa, mortality, pediatrics.
\end{abstract}

http: / / dx.doi.org/10.5546/ aap.2019.eng.128

To cite: Rosanova MT, Mussini MS, Arias AP, Sormani MI, et al. Epidemiological features and risk factors for mortality in Pseudomonas aeruginosa bacteremia in children Arch Argent Pediatr 2019;117(2):128-

a. Department of Epidemiological Control and Infectious Diseases.

b. Department of Microbiology. Hospital de Pediatría SAMIC "Prof. Dr. Juan P. Garrahan," Autonomous City of Buenos Aires.

E-mail address:

María T. Rosanova: M.D.: margris2@yahoo.com.ar

Funding: None.

Conflict of interest: None.

Received: 7-16-2018

Accepted: 11-26-2018
131.

\section{INTRODUCTION}

Pseudomonas aeruginosa (PAE) is a Gramnegative bacillus, an opportunistic nosocomial pathogen. ${ }^{1-2}$ It causes a wide range of infections, especially, in immunocompromised hosts with burn wounds and carriers of cystic fibrosis, among others..$^{2-5}$ Treatment is challenging because of PAE's intrinsic antibiotic resistance and its ability to acquire new resistance mechanisms. PAE bacteremia is related to a high mortality. ${ }^{6}$

\section{OBJECTIVES}

To describe the epidemiological, clinical, microbiological, and evolutionary characteristics of PAE bacteremia. To assess the risk factors for mortality.

\section{MATERIALS AND METHODS}

This was a retrospective, cohort study in children older than 1 month and younger than 18 years admitted between 9/1/2014 and 9/1/2017 with PAE isolation in, at least, one blood culture with or without isolation in a different sterile site.

Data were collected from medical records and included age, sex, origin of bacteremia, underlying disease, prior antibiotic therapy, presence of neutropenia, use of vascular or urinary catheters, invasive procedures in the previous 72 hours, mechanical ventilation (MV) requirement, presence of septic shock at baseline, admission to the intensive care unit (ICU), antibiotic resistance, and clinical course.

\section{Definitions}

Prior exposure to antibiotics was defined as any antibiotic therapy received in the month prior to bacteremia.

Primary bacteremia: positive blood cultures for PAE without other concomitant source of infection.

Secondary bacteremia: positive blood cultures associated with an evident clinical source of infection related to the microorganism, with or without isolation.

Catheter-related bacteremia: isolation of PAE 
in a blood culture from a peripheral vein and in a quantitative or semiquantitative catheter tip culture or from an implantable or semiimplantable catheter without other source of infection other than the catheter.

Shock was defined as a patient with systolic blood pressure below the $5^{\text {th }}$ percentile of the normal range for age, in spite of fluid replacement or inotrope administration.

Deaths during hospitalization of patients diagnosed with PAE bacteremia were considered infection-related.

\section{Microbiological analysis}

Two samples were collected from peripheral blood and / or catheters and the clinical source of infection for culture, as applicable.

Blood samples were inoculated in PF Plus ${ }^{\circledR}$ and FN Plus ${ }^{\circledR}$ bottles (Biomérieux) and incubated in the Bact/Alert 3D ${ }^{\circledR}$ automated system (Biomérieux) during 5 and 7 days, respectively. Microorganisms were identified using a MaldiTof mass spectrometer in the Vitek MS ${ }^{\circledR}$ system (Biomérieux). Sensitivity tests were done using the Kirby-Bauer disc diffusion method and the Vitek $2 \mathrm{C}^{\circledR}$ automated system (Biomérieux). These tests were interpreted in accordance with the recommendations made in the Clinical and Laboratory Standards Institute (CLSI) guidelines. ${ }^{7}$ Carbapenemases were detected using phenotypic methods and confirmed by molecular biology.

Sensitivity tests included the following antibiotics: piperacillin, piperacillin/tazobactam, ceftazidime, cefepime, meropenem and imipenem, gentamicin amikacin, quinolones, and colistin.

Resistance was defined when PAE was resistant to at least one group of active antibiotics. Multidrug resistance was defined when PAE was resistant to three or more families of antibiotics, including antipseudomonal penicillins (piperacillin), cephalosporins (ceftazidime, cefepime), carbapenems, aminoglycosides, monobactams, quinolones and/or polymyxins. Empiric antibiotic therapy was considered adequate if the isolated PAE strain was sensitive to at least one of the antibiotics used.

\section{Statistical analysis}

Data were processed using the Epi-Info version 6.0 software. Continuous outcome measures were expressed as median and interquartile range (IQR); whereas, categorical outcome measures, as absolute quantity and percent relative frequency.

The significance of differences $(p<0.05)$ was assessed based on outcome measure characteristics, with Fisher's exact test or the $\chi^{2}$ test, as applicable. Potential risk factors for mortality due to PAE bacteremia were identified using a univariate analysis.

\section{RESULTS}

In the studied period, the incidence of PAE bacteremia was 3.1 isolations per every 1000 blood cultures (Figure 1).

A total of 100 events were included, which corresponded to 100 patients. All the information required was collected from the medical record review.

Patients' median age was 27 months (IQR: 6-88). Male sex predominated: 52 patients (52\%). An underlying disease was observed in 93 patients $(93 \%)$; blood disease and cancer were the most common ones. Eighty-five patients (85\%) had received antibiotics in the previous month. Invasive procedures were done in $60(60 \%)$, and $81(81 \%)$ had been previously hospitalized. Forty-two patients (42\%) had septic shock upon admission, and $56(56 \%)$ were admitted to the ICU. MV was required in 49 patients (49\%).

Bacteremia was primary in 17 patients $(17 \%)$; catheter-related, in 15 (15\%); and secondary, in 68

FIGURE 1. Flow chart

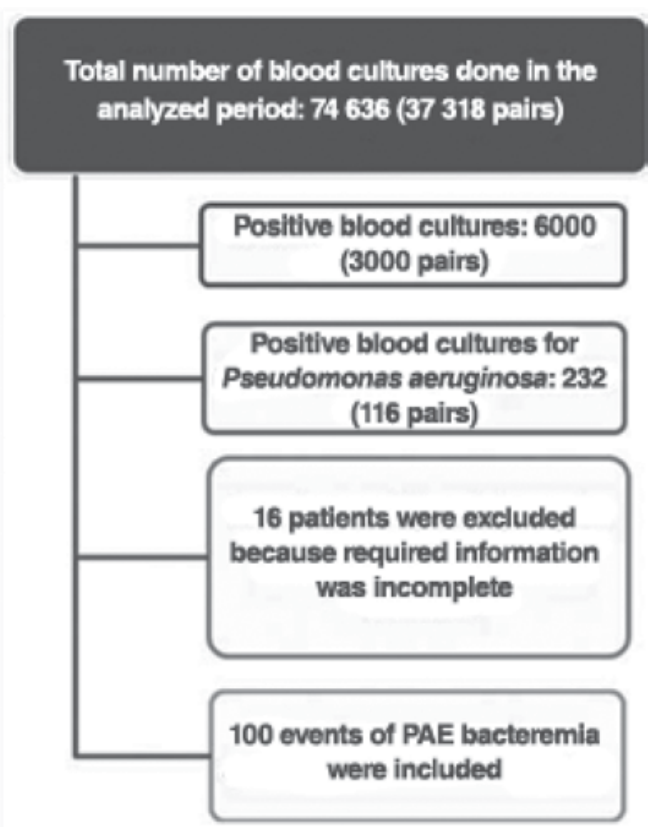

PAE: Pseudomonas aeruginosa. 
$(68 \%)$. The most common source of infection was the skin and mucous membrane, in 21 patients; followed by the lungs, in 20; and the abdomen, in 14 (Table 1). Empiric treatment was adequate in 84 patients $(84 \%)$. Resistance to one or more antibiotic groups was observed in 38 strains, whereas 11 showed multidrug resistance. Fifteen strains were resistant only to carbapenems.

No carbapenemases were detected. All strains were sensitive to colistin (Table 2).

Thirty-one patients died (31\%). Risk factors for mortality in the univariate analysis were septic shock at baseline $(p<0.0005)$, admission to the ICU and MV requirement $(p<0.0001)$, primary bacteremia $(p<0.009)$ or secondary bacteremia $(p<0.003)$, presence of mucocutaneous or pulmonary source of infection $(p<0.004)$, and multidrug resistance $(p<0.01)$ or exclusive resistance to carbapenems $(p<0.01)$.

\section{DISCUSSION}

Bacteremia is a common manifestation of PAE infection; its incidence is approximately 1 event per every 1000 blood cultures, ${ }^{1}$ somewhat lower than that observed in our study.

Immunocompromised patients and those with severe burn wounds, chemotherapy-induced neutropenia or underlying pulmonary disease are

TABLE 1. Patient characteristics and type of bacteremia

\begin{tabular}{lc}
\hline Outcome measure & $\mathbf{n}(\%)$ \\
\hline Mean age in months (IQR) & $27(6-88)$ \\
Underlying disease & $93(93)$ \\
Underlying blood disease and cancer & $42(42)$ \\
Heart disease & $9(9)$ \\
Immunodeficiency & $9(9)$ \\
Burn wound & $7(7)$ \\
Kidney malformation & $7(7)$ \\
Other & $19(19)$ \\
Prior antibiotic use & $85(85)$ \\
Prior hospitalization & $81(81)$ \\
Prior invasive procedure & $60(60)$ \\
Catheter & $82(82)$ \\
Admission to the intensive care unit & $56(56)$ \\
Mechanical ventilation & $49(49)$ \\
Septic shock & $42(42)$ \\
Median length of stay in days (IQR) & $26(14-61)$ \\
Secondary bacteremia & $68(68)$ \\
Primary bacteremia & $17(17)$ \\
Catheter-related bacteremia & $15(15)$ \\
Mucocutaneous source of infection & $21(21)$ \\
Pulmonary source of infection & $20(20)$ \\
Abdominal source of infection & $14(14)$ \\
Mortality & $31(31)$ \\
\hline
\end{tabular}

IQR: interquartile range. more likely to develop PAE infection. ${ }^{6,8,9}$

Although, in some series, an underlying disease in children was a factor correlated to mortality, ${ }^{2,6}$ this was not the case in our study.

Kim et al. ${ }^{10}$ demonstrated that, with an adequate empiric treatment, the presence of neutropenia did not increase mortality, similar to this series.

Cattaneo et al., ${ }^{11}$ assessed PAE bacteremia in cancer patients and reported similar mortality percentages between patients with and without neutropenia.

In our study, catheter-related bacteremia was not a risk factor for mortality, unlike primary or secondary bacteremia. This was similar to what was reported in other studies where catheterrelated bacteremia was not associated with mortality. ${ }^{11,12}$

The secondary sources of infection in PAE bacteremia varied, with predominance of respiratory and mucocutaneous sources, like in our series. ${ }^{10,11}$

Kim et al., ${ }^{10}$ reported, in children with neutropenia, a $30 \%$ and $20 \%$ frequency of respiratory and mucocutaneous source of infection, respectively.

PAE bacteremia had a high rate of morbidity and mortality. High gross mortality rates were associated with patients that required admission to the ICU in some series. ${ }^{11-13}$

In other series, the presence of respiratory distress and MV requirement was an independent risk factor related to mortality. ${ }^{1,13}$

Consistent with other authors, ${ }^{1,10,13}$ the clinical presentation of sepsis was also a predictor of mortality.

In this study, lethality was high and related to admission to the ICU, MV requirement, and the presence of sepsis.

PAE is intrinsically resistant to different antibiotic classes due to the reduction in the patency of its external membrane, the expression of efflux pumps, and the production of antibiotic-

TABLE 2. Antibiotic sensitivity (100 strains)

\begin{tabular}{lc}
\hline Antibiotic & Resistant (n) \\
\hline Antipseudomonalpenicillins & 16 \\
Antipseudomonalcephalosporins & 14 \\
Carbapenems & 30 \\
Monobactams & 7 \\
Aminoglycosides & 17 \\
Quinolones & 11 \\
Polymyxins & 0 \\
\hline
\end{tabular}


inactivating enzymes, and is also capable of acquiring new resistance mechanisms through mutations. Prior antibiotic use favors the development of antibiotic resistance, which is consistent with the outcomes of this study. ${ }^{1,4,6,9,10}$

The consequences of resistance and the impact of an inadequate treatment on the course of patients with PAE bacteremia are not entirely clear. Carbapenems have been considered firstline agents for the treatment of severe PAE infections; however, resistance has increased..$^{10-14}$

Some studies have suggested that the antibiotic resistance of PAE was not a predictor of mortality. ${ }^{10}$ Other authors have reported that carbapenem-resistance would be associated with a higher mortality than from infections caused by sensitive strains. ${ }^{11,12}$

In other series, multidrug resistance was independently associated with an inadequate therapy, which was a major predictor of mortality, synergistic with the severity of the underlying disease. ${ }^{10-14}$

Carbapenem resistance and multidrug resistance were statistically significant predictors of mortality in our series. A high percentage of our patients received an adequate empiric therapy, which would explain the fact that the latter outcome measure did not show a statistically significant relation to mortality.

\section{CONCLUSIONS}

PAE bacteremia predominated among patients with an underlying disease. Mortality was high and risk factors included septic shock at baseline, admission to the ICU, MV requirement, presence of primary bacteremia or non-catheterrelated secondary bacteremia, presence of mucocutaneousor pulmonary source of infection, and multidrug resistance or exclusive resistance to carbapenems.

\section{REFERENCES}

1. Yang MA, Lee J, Choi EH, Lee HJ. Pseudomonas aeruginosa bacteremia in children over ten consecutive years: analysis of clinical characteristics, risk factors of multi-drug resistance and clinical outcomes. J Korean Med Sci. 2011;
26(5):612-8.

2. Grisaru-Soen G, Lerner-Geva L, Keller N, Berger H, et al. Pseudomonas aeruginosa bacteremia in children: analysis of trends in prevalence, antibiotic resistance and prognostic factors. Pediatr Infect Dis J. 2000; 19(10):959-63.

3. Magiorakos AP, Srinivasan A, Carey RB, Carmeli Y, et al. Multidrug-resistant, extensively drug-resistant and pandrug-resistantbacteria: an international expert proposal for interim standard definitions for acquired resistance. Clin Microbiol Infect. 2012; 18(3):268-81.

4. Zhang Q, SmithJC, Zhu Q, Guo Z, et al. A five-year review of Pseudomonas aeruginosa bacteremia in children hospitalized at a single center in southern China. Int J Infect Dis. 2012; 16(8):e628-32.

5. Bauer A, Kirby W, Sherris J, Turck M. Antibiotic susceptibility testing by a standardized single disk method. Am J Clin Pathol. 1966; 45(4):493-6.

6. Dantas RC, Ferreira ML, Gontijo-Filho PP, Ribas RM. Pseudomonas aeruginosa bacteraemia: independent risk factors for mortality and impact of resistance on outcome. J Med Microbiol. 2014; 63(Pt 12):1679-87.

7. Clinical and Laboratory Standards Institute. Performance standards for antimicrobial susceptibility testing. $26^{\text {th }}$ ed. CLSI supplement M100S. Wayne, PA: CLSI; 2016.

8. Hu Y, Li L, Li W, Xu H, et al. Combination antibiotic therapy versus monotherapy for Pseudomonas aeruginosa bacteraemia: a meta-analysis of retrospective and prospective studies. Int J Antimicrob Agents. 2013; 42(6):4926.

9. Kang CI, Kim SH, Kim HB, Park SW, et al. Pseudomonas aeruginosa bacteremia: risk factors for mortality and influence of delayed receipt of effective antimicrobial therapy on clinical outcome. Clin Infect Dis. 2003;37(6):74551.

10. Kim YJ, Jun YH, Kim YR, Park KG, et al. Risk factors for mortality in patients with Pseudomonas aeruginosa bacteremia; retrospective study of impact of combination antimicrobial therapy. BMC Infect Dis. 2014; 14:161.

11. Cattaneo C, Antoniazzi F, Casari S, Ravizzola G, et al. $P$. aeruginosa bloodstream infections among hematological patients: an old or new question? Ann Hematol. 2012; 91(8):1299-304.

12. Schechner V, Gottesman T, Schwartz O, Korem M, et al. Pseudomonas aeruginosa bacteremia upon hospital admission: risk factors for mortality and influence of inadequate empirical antimicrobial therapy. Diag Microbiol Infect Dis. 2011; 71(1):38-45.

13. Kim HS, Park BK, Kim SK, Han SB, et al. Clinical characteristics and outcomes of Pseudomonas aeruginosa bacteremia in febrile neutropenic children and adolescents with the impact of antibiotic resistance: a retrospective study. BMC Infect Dis. 2017; 17(1):500.

14. Jeong SJ, Yoon SS, Bae IK, Jeong SH, et al. Risk factors for mortality in patients with bloodstream infections caused by carbapenem-resistant Pseudonoma aeruginosa: Clinical impact of bacterial virulence and strains on outcome. Diagn Microbiol Infect Dis. 2014; 80(2):130-5. 